\title{
Object Recognition Based Power Plant Management System in Smart Grid using Smart Device
}

\author{
Young-Jae Lee*, Kyeong-Jin Ban*, Kyoung-Wook Park**, Seung-Heon Song***, Eung-Kon Kim * \\ *Department of Computer Science, Sunchon National University \\ **Division of Culture Contents, Chonnam National University \\ ***Green Energy Institute
}

\{leeyoungjae, multwave, kek\}@sunchon.ac.kr, zergadiss73@chonnam.ac.kr, fsaa@gei.re.kr

Corresponding Author: Eung-Kon Kim

\begin{abstract}
Today, the world is facing diverse problems such as energy saving, climate change and economic growth etc. To overcome those problems, an introduction of a smart grid is considered to be dispensable for satisfying future energy requirements such as rationalization of power consumption, stimulation of lowcarbon energy technologies through less consumption of fossil fuel, greater use of renewable energy like photovoltaic and wind power, and a need of next-generation growth engine. In this paper, an object recognition based smart grid photovoltaic power facility management system is proposed. Using the proposed system, maintenance and repairs required due to regular inspection or faults can be carried out more efficiently in a remote site. Additionally, the proposed system takes an object recognition based technology instead of using QR code when recognizing the facility. It implies that this approach can be applied straight away to an existing system because it does not require any need of attaching an additional equipment to an existing smart grid power facility. Furthermore, the proposed system applies a markerlessbased object recognition technique. Therefore it is expected that this service can be easily extended to recognizing other types of facilities such as smart grid transmission and distribution facilities in the future.
\end{abstract}

Keywords - Smart Grid, Power plant facility management, Smart Phone, Location Based Service

\section{INTRODUCTION}

Currently, the world is facing a variety of challenges such as energy saving, climate change and economic growth etc. To resolve the problems, an introduction o`f a smart grid is considered to be dispensable, from the perspective of power consumption rationalization, low-carbon energy technologies stimulation through less consumption of fossil fuel, greater use of renewable energy like photovoltaic and wind power, and next-generation growth engine[1-3]. A smart grid is a next-generation intelligent electric power transmission network that enables to easily make use of renewable energy in an existing power network and optimizes energy efficiency [4] [5].

In a smart grid, energy generation facilities are varied and located in lots of scattered places. Photovoltaic and wind power facilities are installed in residential houses, buildings, parking lots and large-scale power plant located in public facilities. As of products used in these facilities, a wide variety of types and specifications are available. Therefore, a site manager is required to visit the facility in person in order to check the facility because the products and specifications used in the facility can be varied. Moreover, time duration and accuracy for the checking can differ depending on a manager's capability.

For achieving better maintenance and checking, it is necessary to provide various information including location of the facilities, product information, power generation capacity, specification, measurement data, hardware diagram and maintenance history to the manager.

In this paper, an object recognition based smart grid photovoltaic power facility management system is proposed. This system is designed to provide information about smart grid power facility using smartphone. Currently, most of management systems are developed using QR code and based on PC. Hence, a manager can monitor states of facility and information related to power generation only through a designated computer. Additionally, maintenance can be done by obtaining data from QR code. In contrast, the proposed methodology enables more efficient maintenance, since maintenance can be conducted even in a remote site in either case of regular inspection or repair by faults. Instead of using QR code, the proposed approach takes an object recognition based technology. It implies that this approach can be applied straight away in an existing system because it does not require any need of attaching an additional equipment to an existing smart grid power facility. In addition, the proposed system has advantage over a traditional system such that the service provided by the proposed system can be easily extended to other types of facilities like smart grid transmission and distribution facilities because it applies a markerless-based object recognition technique. 


\section{RELATED WORKS}

Existing smart grid power facility management systems are divided into two categories: GIS-based and QR-code based system.

\section{A. GIS-based Smart Grid Data Visualization System.}

Open source GIS-based smart grid data visualization system is a system designed to visually display smart grid power plant location, consumed power, voltage, current and total amount of power consumed by a region. Those data are presented on a map by means of Google Earth[6-7].

Though this system provides some information including power plant location, power consumption and voltage, it has disadvantage such that available information is restricted. Specifically, this system is not capable of providing detailed information such as products used in power facility, specifications, maintenance history and drawings.

\section{B. QR code Service (Smart M2M Service)}

Figure 1 shows how QR code-based technology works. Power facility is recognized using QR code. Then user connects to M2M server using smartphone. Accessing to M2M server, user can get information such as past history and states regarding various power facilities of distributing board, motor controlling board, switchboard and photovoltaic power generation facility [8].

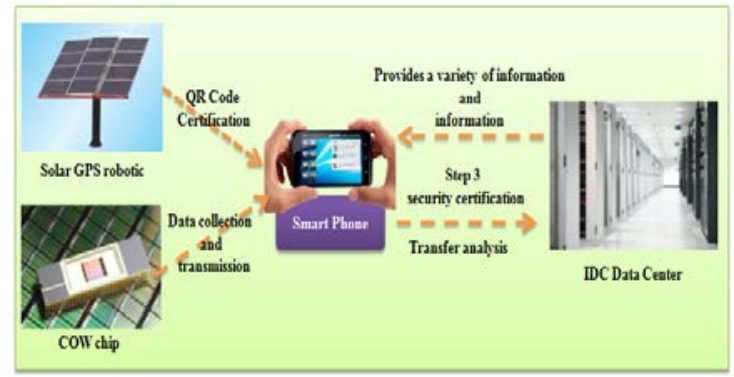

Figure 1. QR code service (Smart M2M Service)

Using QR code service, user can acquire information about power facility through smartphone in realtime. However, QR code must be attached in advance. Even though QR code is attached completely, QR code might get damaged by external contamination. Once the QR code gets damaged, it is hard to get accurate information.

\section{OBJECT RECOGNITION-BASED FACILITY MANAGEMENT SYSTEM FOR SMART GRID PHOTOVOLTAIC POWER PlANT.}

In this paper, object recognition- based facility management system for smart grid photovoltaic power plant is proposed. This system mainly consists of two components: (1) server system which provides information such as current state, maintenance, inspection and management history about facility, and design drawing, and (2) a facility management applica- tion which is based on an object recognition technique. Overview of this system is illustrated in Figure 2.

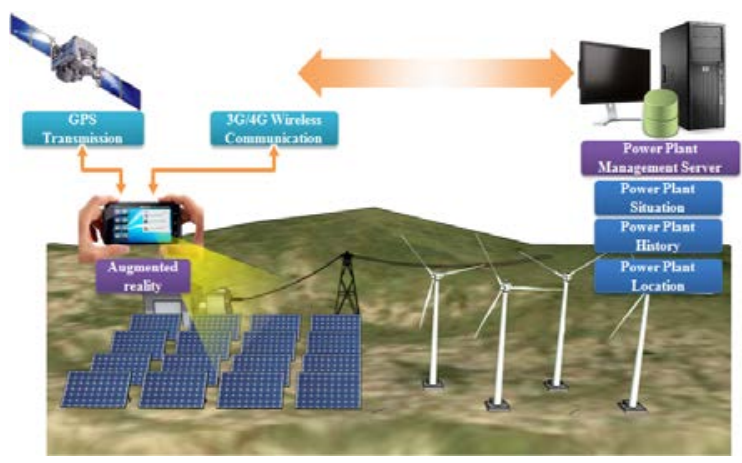

Figure 2. Overview of an object-based facility management system for smart grid power plant.

\section{A. Server System for Smart Grid Power Facility Manage- ment}

A server system is implemented on top of WAS(Web Application Server) in order for easy cooperation with smartphone. The server system offers information on power facility such as current state, maintenance/inspection history, and design diagram.

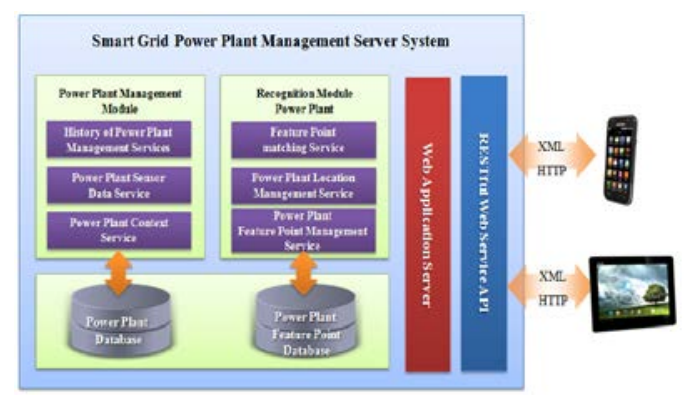

Figure 3. Server System Diagram of smart grid facility management system.

REST API is used for implementing system, since it offers platform- independent interface. Hence our system can effectively support diverse types of smartphones and table PCs. Furthermore, the use of REST API enables perfect integration with smartphone applications and scaling up.

\section{B. Location-based Smart Grid Power Facility Guidance Service.}

Location-based smart grid power facility guidance service navigates an accessing path to a power generation facility using Google Maps API. Since a power generation facility tends to be scattered across a large area, this guidance service aids the site operator to move to next place efficiently and promptly. Figure 4 shows an overall architecture of smart grid power facility guidance system. 


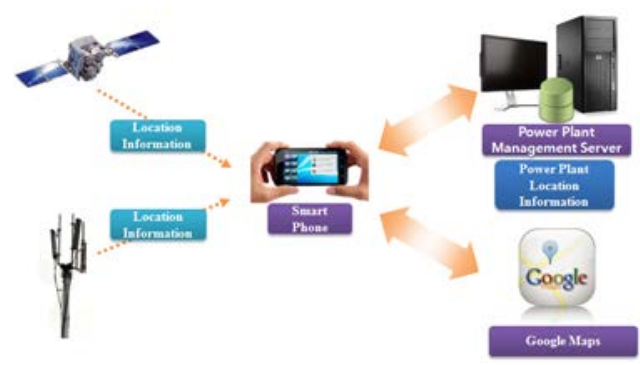

Figure 4. Architecture of Smart Grid Power Facility Guidance System Conclusions

\section{Smart Grid Power Facility Recognition}

An object recognition-based smart grid power facility management system is necessary to recognize a power generation facility. To do this, the server stores the data such as the location where the facility is located and its images. Once the server recognizes the facility as requested by the site operator, it provides the correspondent data to the operator. The process how the system recognizes and provides an appropriate data is as shown in figure 5 .

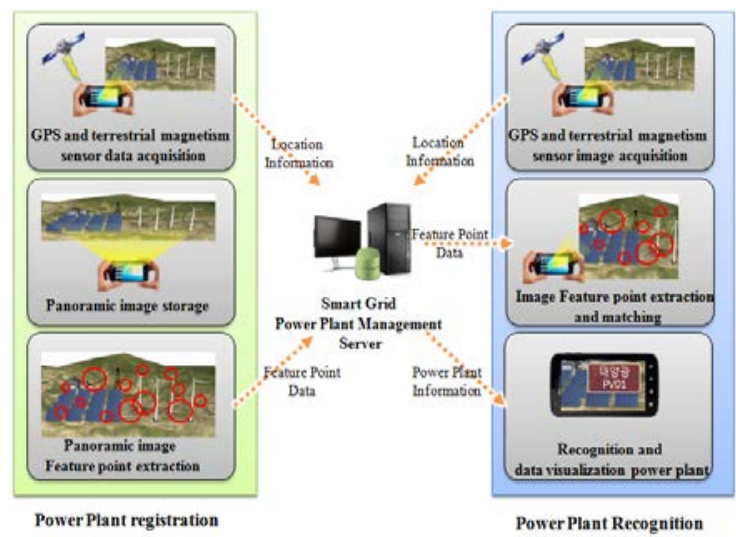

Figure 5. Process how the proposed system recognizes the power facility

When registering the data regarding smart grid power facility, first step is to take pictures of view around the facility as panoramic images. Next step is to extract feature points from these panoramic images and to store them to database. When an operator arrives at the site, he takes a picture around the facility in an adjacent area using his smartphone. Then feature points are extracted from the image taken by a smartphone camera. Once the feature points are obtained, a matching operation is carried out by comparing the extracted feature points with those of panoramic images stored in database. As a result of the matching operation, the system now obtains information and history about the matched facility.

In this paper, when extracting feature points, we use the Speeded Up Robust Feature(SURF) algorithm to allow the correct recognition even if the panoramic image taken by smartphone has been rotated or with different size. Figure 6 shows photos before and after detecting feature points using SURF algorithm.

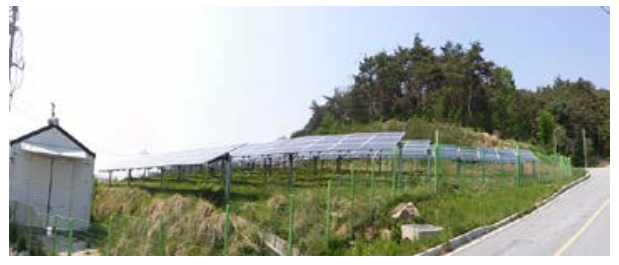

(a)Panoramic Image

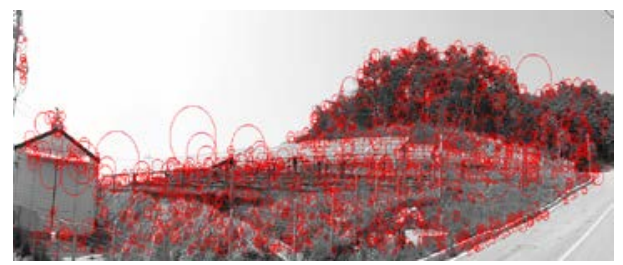

(b)Detecting Feature Points

Figure 6. Detecting Feature Points using SURF to recognize the power facility

After extracting feature points from an input image sent by a camera and transferring them to a server, the server carries out comparison of these feature points with respect to those stored in database. When finding the panoramic image with best matching rate, the server sends relative information back to smartphone. Figure 7 is a feature points matching test result using SURF algorithm.

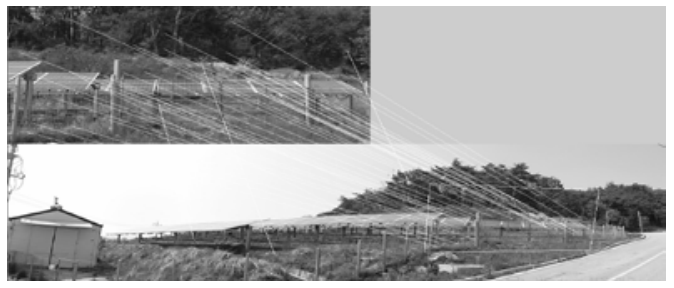

(a)Feature Points Matching 1

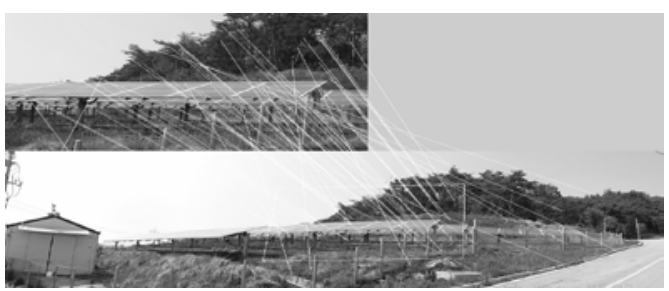

(b)Feature Points Matching 2

Figure 7. Smartphone Application for Object-based Power Facility Management

Main functionalities of smartphone application implemented in this paper are as follows: (1)recognize power facility through an image taken by smartphone, (2)manage the power facility by making use of data sent by a server and (3)obtain latitude, longitude and location information provided by Google Maps. 
Smartphone application for smart grid power facility management system is executed as shown in figure 8. Figure 8 presents user's login screen. In this screen, a site manager enters user id and password. The application gets id and password and then sends them to a server. The server performs authentications of the user.
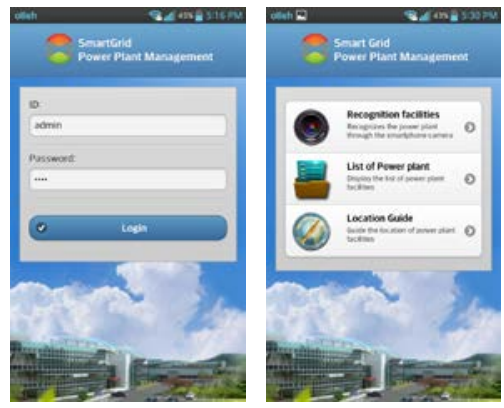

Figure 8. Screen shot of user login and main menu

The application presents three main menus: recognize power facility, view power facilities list, and navigate power facility location. When the user selects one of menus by touching it, correspondent function would be invoked.

Figure 9 is Power Facility Recognition. as for a recognition of power facility, an image taken by a Smartphone camera is used to extract feature points of the facility. The extracted data is sent to a server. (a) Camera Image

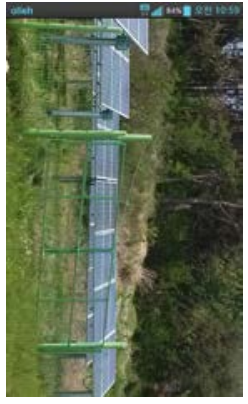

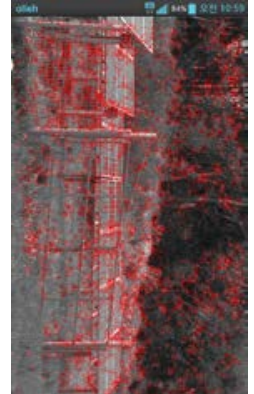

(b) Feature Points Extraction
Figure 9. Power Facility Recognition

Once the server receives the feature points and GPS location data sent by the Smartphone application, the server carries out a matching operation by searching data stored in database. When the matching is successfully completed, the server returns the correspondent data back to the smartphone application as shown in figure 10 .

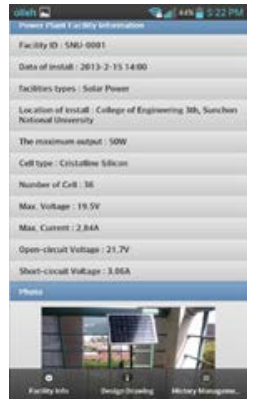

(a) Power Facility Information

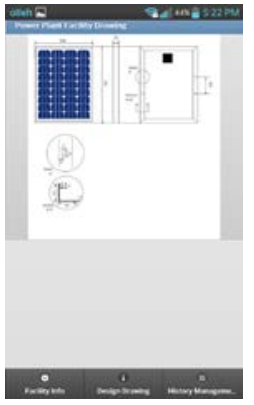

(b) Power Facility Drawing
Figure 10. Power facility information/List of all the power facilities

Figure 11 shows how power facility location guidance service provides the locations of power facilities in an adjacent area together with current position of an operator.

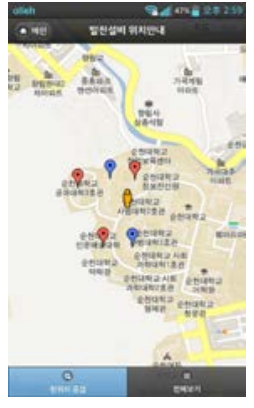

(a) Current location of the operator

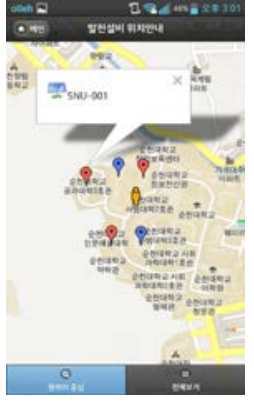

(b) Facilities in an adjacent area
Figure 11. Power Facility Location Guide

At the screen of the guidance service, if the user clicks one of facilities as expressed on Google Maps, the user can see the details about the facility such as its type. There are three kinds of marker used. A marker @िdenotes current position of the operator. A marker $\odot$ denotes a location of wind power facility and $\odot$ points to a location of power facility

\section{IV.CONCLUSIONS}

In this paper, we proposed and developed an object recognition-based system which aids an site operator to recognize a facility and to obtain details about the recognized facility through a wireless device like smartphone and tablet PC. To recognize the facility, this study suggests to apply GPS, geomagnetic sensor and camera embedded in a smartphone to the proposed system. Once the facility has been recognized, the system offers details about the facility such as hardware diagrams, sensor data and history to an operator working at a remote site in a realtime basis.

Using the proposed system, the site manager can effectively operate maintenance tasks in either case of regular inspection or repair by faults.

Additionally, the system takes an object recognition-based technology instead of QR code. It implies that this approach 
can be applied straight away to an existing system because it does not require any need of attaching an additional equipment to an existing smart grid power facility. In addition, the proposed system has advantage over a traditional system. It applies a markerless object recognition technique so that this service might be easily extended to recognizing other types of facilities such as smart grid transmission and distribution facilities.

As a future work, the proposed system can be extended to realistic visualization of design drawing and detailed information about the power facility using markerless-based augmented reality technology. In addition, we will explore more information suitable for a site's situation. The future visualization system will improve to dealing with those further information in realtime.

\section{ACKNOWLEDGMENT}

This research was financially supported by the Ministry of Education (MOE) and National Research Foundation of Korea(NRF) through the Human Resource Training Project for Regional Innovation (No. 2013H1B8A2032217).

\section{REFERENCES}

[1] V. C. Gungor, D. Sahin, T. Kocak, S. Ergut, C. Buccella, C. Cecati, and G.P. Hancke, "Smart Grid Technologies: Communication Technologies and Standards," IEEE Transactions on Industrial Informatics, vol. 7, no.4, pp. 529-539, Nov. 2011.

[2] Zhong Fan, Parag Kulkarni, Sedat Gormus, Costas Efthymiou, "Smart Grid Communications: Overview of Research Challenges, Solutions, and Standardization Activities", IEEE Communications Surveys \& Tutorials, Vol. 15, No. 1, pp. 21-37, 2013

[3] Nejad, M.F, Saberian, A.M, Hizam, H. Radzi, M.A.M, "Application of Smart Power Grid in Developing Countries", Power Engineering and Optimization Conference (PEOCO) 2013 IEEE 7th International, pp. $427-431,2013$

[4] Xi Fang, Satyajayant Misra, Guoliang Xue,” Smart Grid - The New and Improved Power Grid: A Survey", Communications Surveys \& Tutorials, IEEE Volume.14 , Issue. 4, pp. 944-980,2012

[5] Petinrin. J.O, Shaaban, M, "Smart Power Grid: Technologies and Applications”, Power and Energy (PECon), 2012 IEEE International Conference on, pp. 892 - 897, 2012.

[6] Dao Viet Nga, Do Nguyet Quang, "Open Source GIS-Based System for Displaying Data in Smart Grid”, 2012 International Conference on Smart Grid Systems (ICSGS 2012) IPCSIT vol.45, pp.17-21, 2012.

[7] Ethan Boardman, "The Role of Integrated Distribution Management Systems In Smart Grid Implementations”, Power and Energy Society General Meeting, 2010 IEEE, pp.1-6, 2010.

[8] Kdpower website. [Online]. Available: http://www.kdpower.co.kr/.

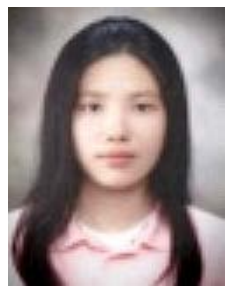

Young-Jae Lee

Young-Jae Lee received the B.S. degree from Korea Sunchon National University, Korea, in 2012, She is currently a M.S. student in computer science at the Sunchon National University, Korea. Her current research interests include augmented reality, image processing, computer graphics.

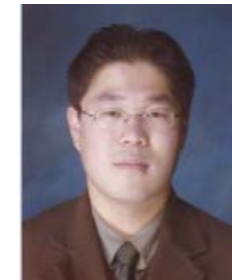

\section{Kyeong-Jin Ban}

Kyeong-Jin Ban received the B.S., M.S., and Ph.D. degrees in computer science from Sunchon National University, Korea, in 2003, 2005, 2011, respectively. His current research interests include computer graphics, wireless sensor network, and augmented reality.

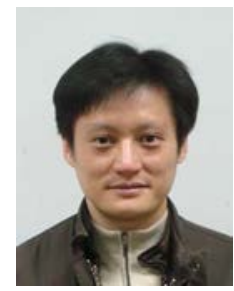

Kyoung-Wook Park

Kyoung-Wook Park received the B.S. degree from Sunchon National University, Suncheon, Korea, in 1997, his M.S degree from department of computer science and statistics, Chonnam National University, Gwangju, Korea, in 1999, his Ph.D. degree from department of computer science, Chonnam National University, Gwangju, Korea, in 2004. His current research interests are parallel and distribution processing, graph theory, theory of computation, bioinformatics and augmented

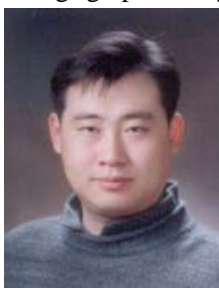
reality.

\section{Seung-Heon Song}

Seung-Heon Song received the M.S., and Ph.D. degrees in computer science from Sunchon National University, Korea, in 2000, 2006, respectively. Since 2010, he has been working as general manager at the Green Energy Institute, Mokpo, Korea. His current research interests include renewable energy, computer graphics and ubiquitous computing.

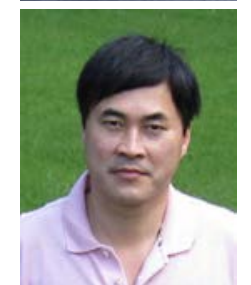

Eung-kon Kim(Corresponding Author)

Eung-kon Kim received the B.S. degree from Chosun University, Gwangju,, Korea, in 1980, his M.S degree from department of electronics, Hanyang University, Seoul, Korea, in 1987, his Ph.D. degree from Chosun University, Gwangju, Korea, in 1992. His current research interests are computer vision, virtual/augmented reality, image processing, and compute graphics. Currently he is a professor in department of computer engineering, Sunchon National University, Korea. 